\title{
Archéopages
}

Archéopages

Archéologie et société

46 | 2018

Maisons

\section{Les fermes gauloises et antiques de la moyenne vallée de l'Oise}

Des établissements ruraux sans maison?

The Gallic and ancient farms of the middle valley of the Oise. Rural settlements without houses?

Las granjas galas y antiguas del valle medio del río Oise: ¿instalaciones rurales sin vivienda?

\section{Denis Maréchal}

\section{OpenEdition}

Journals

\section{Édition électronique}

URL : https://journals.openedition.org/archeopages/3730

DOI : 10.4000/archeopages.3730

ISSN : 2269-9872

Éditeur

INRAP - Institut national de recherches archéologiques préventives

Édition imprimée

Date de publication : 1 décembre 2018

Pagination : 16-25

ISSN : 1622-8545

Référence électronique

Denis Maréchal, « Les fermes gauloises et antiques de la moyenne vallée de l'Oise », Archéopages [En ligne], 46 | 2018, mis en ligne le 01 décembre 2020, consulté le 02 juin 2021. URL : http://

journals.openedition.org/archeopages/3730 ; DOI : https://doi.org/10.4000/archeopages.3730 


\section{Les fermes gauloises et antiques de la moyenne vallée de l'Oise}

Des établissements ruraux sans maison?

Denis Maréchal Inrap

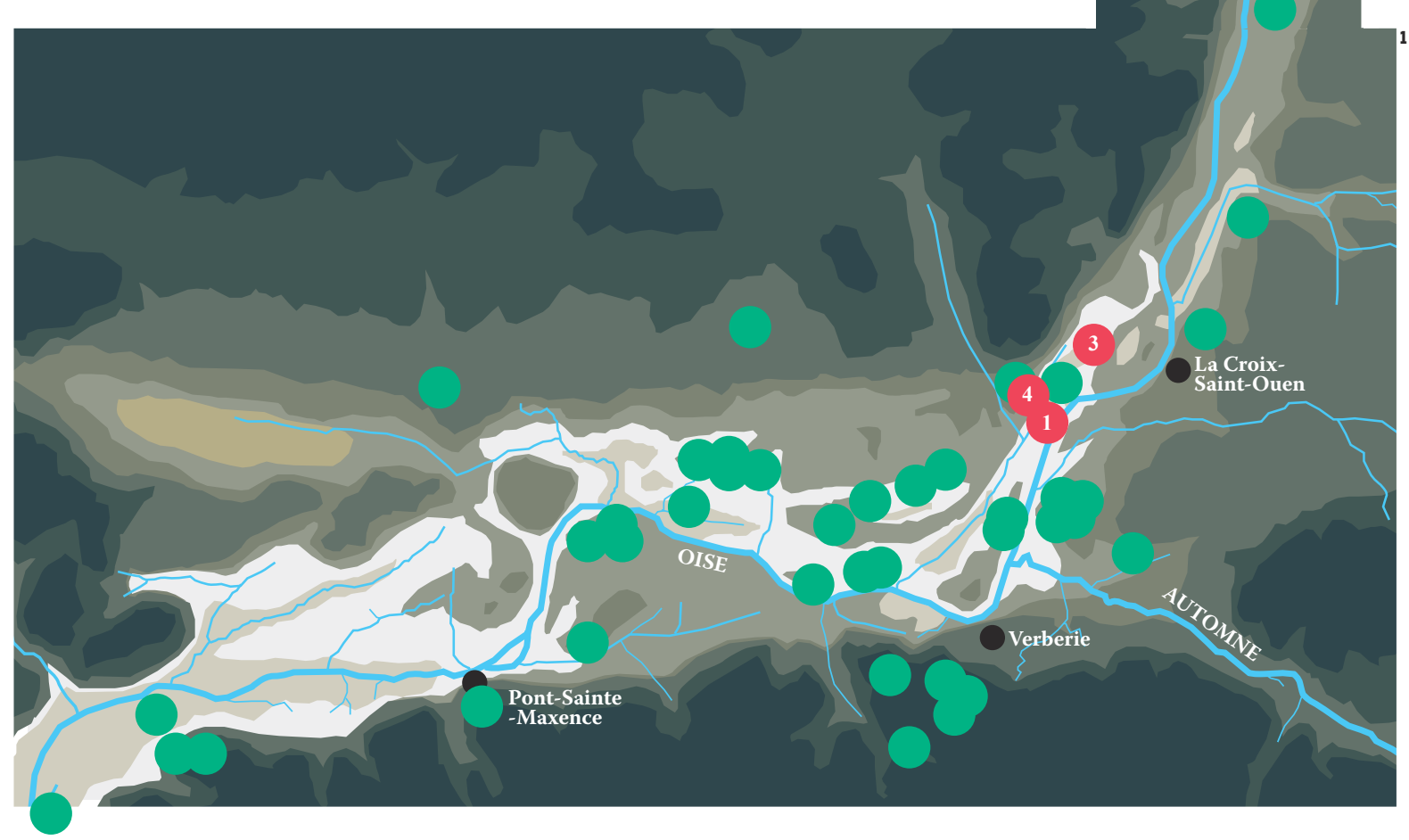

\begin{tabular}{l|l|l|l|l|l|l} 
inf. à $30 \mathrm{~m}$ & 30 à 31,24 & 31,25 à 32,49 & 32,50 à 34 & 35 à & 49 & à 74
\end{tabular}

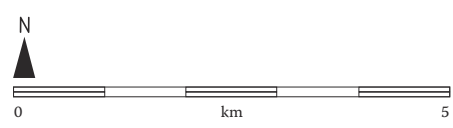


Les multiples fouilles préventives réalisées dans la portion de vallée de l'Oise située au sud-ouest de la ville de Compiègne et ses rebords de plateau ont permis d'appréhender plus de 680 ha de 1987 à 2017 et une soixantaine de sites datés entre La Tène moyenne/finale ( $\mathrm{III}^{\mathrm{e}}$ siècle avant notre ère) et la période antique ( $\mathrm{III}^{\mathrm{e}}$ siècle) (Malrain, Maréchal, 2012 ; Malrain et al., 2017) [ill. 1]. Parmi la trentaine d'habitats fouillés ou diagnostiqués, une majorité ne livrent que de petits édifices sur trous de poteau, parfois des nuages de ces mêmes structures et, plus rarement, des fondations sur solins ou des sablières. Alors que ce sont les structures de stockage sur 4 ou 6 poteaux qui prédominent (Pinard et al., 1999), le sous-effectif des grandes constructions, donc de possibles maisons, nous a paru un fait à interroger.

Pourquoi les bâtiments d'habitation de ces unités rurales ne sont-ils pas conservés ? Nos recherches nous avaient déjà mené à établir le fait que les établissements ruraux en plaine alluviale occupent des emplacements correspondant à des éminences où ils sont à l'abri des crues annuelles et décennales (Maréchal, 2005, p. 180 ; Malrain, Pinard et Maréchal, 2006, p. 47). La faible profondeur de découverte des vestiges (entre 0,30 et $0,50 \mathrm{~m}$ sous la terre végétale) montre que l'érosion, et en particulier celle due aux labours récents, est importante. Sur les rebords de plateau, l'action érosive semble également forte et l'épaisseur de sédiment contemporain à peu près comparable (autour de o,50 m). Certains édifices sont néanmoins perçus dans des conditions similaires de « conservation ». L'explication érosive ne peut donc que partiellement expliquer la sous-représentation des bâtiments principaux. L'hypothèse du recours à des architectures particulières peu ancrées dans le sous-sol pourrait résoudre la question : architectures fondées sur des sablières basses ou tranchées de fondation pour les périodes les plus anciennes et sur solins pour les phases du $\mathrm{II}^{\mathrm{e}} / \mathrm{III}^{\mathrm{e}}$ siècle. Les exemples suivants présentent les moyens de mieux repérer les vestiges qui indiquent la présence de ces maisons.

\section{Les sites laténiens}

Pour la période laténienne, la détection des habitats peut s'appuyer sur les modes de rejets. Les durées d'occupation courtes, réduites à une ou deux générations, sont marquées par des rejets massifs dans les fossés définissant la majorité des établissements. Depuis longtemps, François Malrain a mis en évidence ce phénomène récurrent pour cette période (Malrain, 1990 et 2000). Les dépôts principaux sont très probablement en lien avec de possibles maisons et situés au plus proche des bâtiments. L'étude des cartes de répartition de différentes catégories de matériel et de la présence d'objets liés à des activités distinctes permet de déduire la nature des édifices et/ou leur statut (Malrain 2006, p. 75 et 85).
Dans la plaine alluviale, l'établissement du Petit Pâtis à Rivecourt est daté de La Tène $\mathrm{C}_{2} / \mathrm{D}_{1}$ soit de la seconde moitié du $\mathrm{II}^{\mathrm{e}}$ et du début du $\mathrm{I}^{\text {er }}$ siècle avant notre ère ${ }^{\mathbf{1}}$ (Maréchal, 2014). Dans l'enclos - partiellement dégagé - qui couvre $4800 \mathrm{~m}^{2}$, il a été distingué de façon certaine huit constructions, dont sept de moins de $10 \mathrm{~m}^{2}$ et une de $4.2 \mathrm{~m}^{2}$ [ill. 2]. Lors de l'intervention archéologique, les fossés ont été fouillés ou curés avec un enregistrement linéaire et par couche stratigraphique. Toutes les catégories de mobilier (céramique, lithique, archéozoologique et métallique) ont été inventoriées. La dispersion des artefacts est peu variable : c'est toujours la moitié nord qui regroupe l'essentiel des mobiliers quelle que soit leur nature, et en particulier l'angle nordest [ill. 3]. On note cependant certains décalages. Ainsi, au niveau de la principale concentration, l'enregistrement par strate permet d'opérer des distinctions selon les phases. Durant les trois périodes d'occupation, c'est le fossé nord de l'enclos (7004) qui concentre les plus gros volumes, suivi de la moitié septentrionale du fossé ouest (7048) [ill. 4]. Mais aux phases ancienne et médiane, les principales concentrations se situent derrière le petit enclos interne $(7115 / 7152)$. Un deuxième amas est noté dans la partie nord du fossé ouest (7048). Dans l'ultime comblement, un important ensemble se trouve dans la partie ouest du fossé nord (7004) alors que dans le fossé ouest (7048), les amas sont plus dispersés que précédemment. On en déduit qu'un habitat principal existe au niveau du petit enclos interne $(7115 / 7152)$, bien qu'on n'en trouve pas de traces au sol, et ce sur toute les phases d'occupation. En revanche, le vaste édifice de $4.2 \mathrm{~m}^{2}$, à l'ouest, ne serait construit que lors de la dernière phase. S'agit-il aussi d'un habitat? Au niveau du fossé ouest (7048), il existerait également des déplacements des constructions (nouveaux édifices reconstruits avec un décalage de quelques mètres) selon les moments, certains ne laissant pas de traces.

Sur le rebord de plateau, la ferme du Bois de Plaisance à Venette ${ }^{2}$ date de La Tène D1 - soit entre 120 et 80 avant notre ère - et l'enclos couvre $8450 \mathrm{~m}^{2}$ (Maréchal, 2011a et b). Intégralement décapé, le site compte sept constructions concentrées dans deux enclos emboîtés de 4. $030 \mathrm{~m}^{2}$ [ill. 5]. Aux quatre structures classiques de stockage sur quatre ou six poteaux, il faut ajouter des constructions plus rares. L'une évoque une ossature de «tour », car elle est constituée de neuf trous de poteau d'un diamètre moyen de 1,05 $\mathrm{m}$ et d'une profondeur de 0,25 m (dimensions nettement supérieures aux autres trous de poteau du site, en largeur et en profondeur), concentrés sur une surface de $14 \mathrm{~m}^{2}$. Un exemple assez proche de « tour » a été identifié à Bazoches-sur-Vesles (Aisne) (Gransar et Pommepuy, 2005). L'interprétation des deux derniers édifices de Venette nécessite le recours aux rejets : le bâtiment 128, proche de l'entrée orientale d'un des
2. Fouillée en 2004, sous la responsabilité de l'auteur. 
2. Le Petit Pâtis à Rivecourt,

répartition du mobilier

céramique et interprétation

des bâtiments. Les

principales concentrations se

situent autour du petit enclos

interne - vide de trace

d'édifice - alors qu'une série

de constructions se trouvent

plus à l'ouest mais génèrent

nettement moins de déchets.

$\stackrel{\infty}{=}$

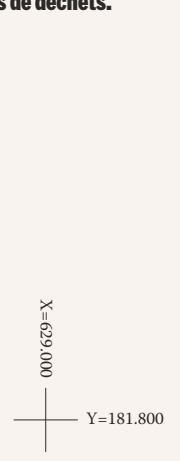

Mobilier céramique (exprimé en g)

$\begin{array}{ll}\circ & \text { inf à } 118 \\ 118 \text { à } 459 \\ 460 \text { à } 969 \\ 970 \text { à } 1499 \\ \text { sup. à } 1500\end{array}$

unité d'habitat?

silo

forge?

zone de St.

grenier, remise?
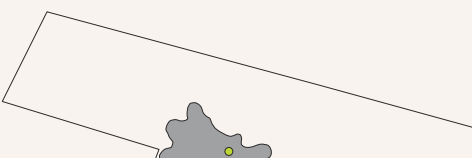
3. Rivecourt, répartition de
la faune, du grès et du torchis.
Si le petit enclos concentre
toujours une majorité
des corpus, d'autres amas
ressortent, en particulier
à l'ouest, avec des décalages
selon les matériaux.
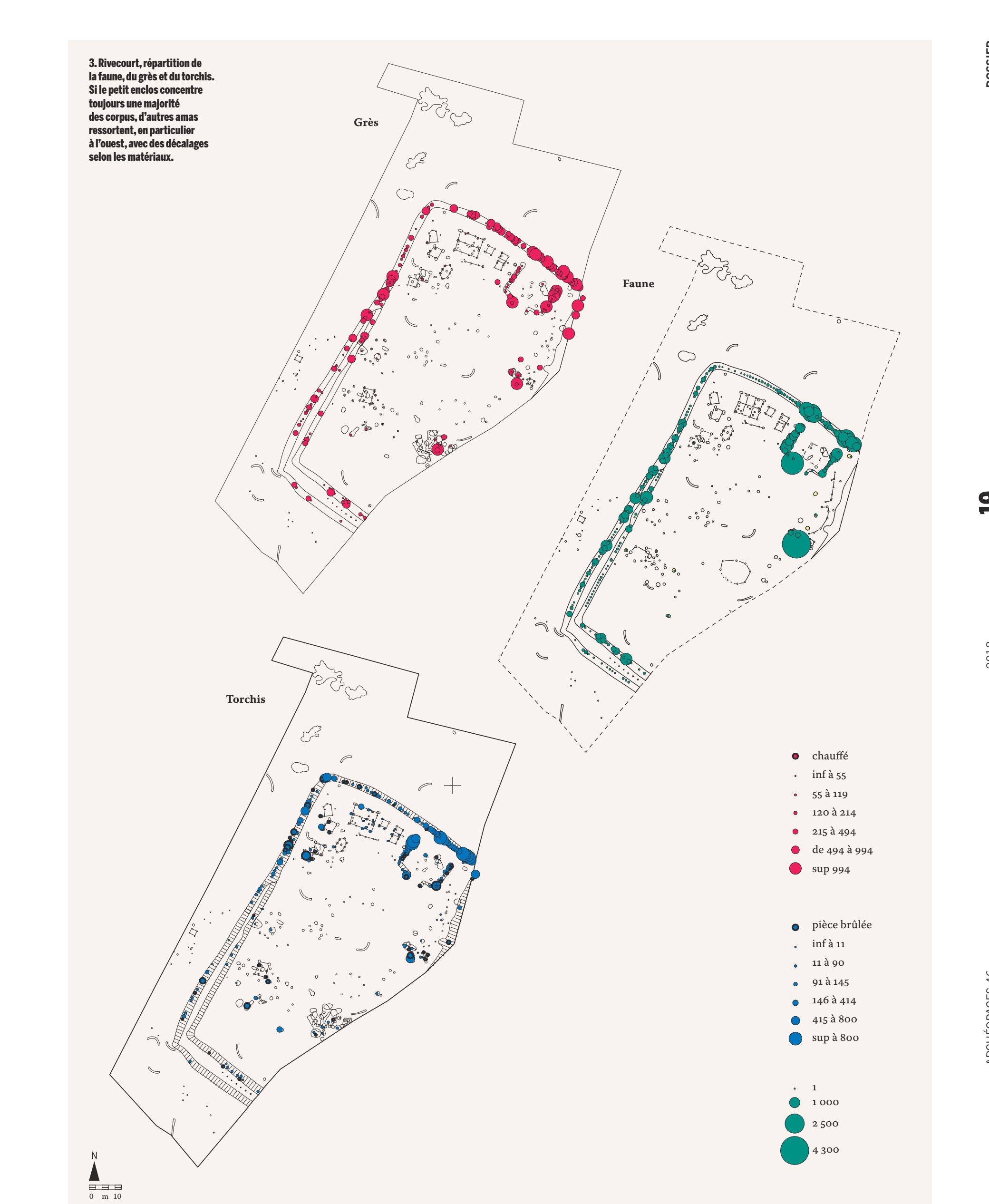

\section{요}


4. Rivecourt, répartition de

la céramique par couche

stratigraphique. Pour le fossé

nord (7004), les amas

principaux dans les couches

inférieures et moyennes se

situent autour du petit enclos

interne, alors que, durant

la dernière phase, une autre

concentration ressort au

niveau du grand bâtiment.

Couche 1 (sup.)

Pour le fossé ouest (7048)

il existe également des

décalages entre les trois

phases qui peuvent

témoigner du glissement de

constructions visibles ou non.

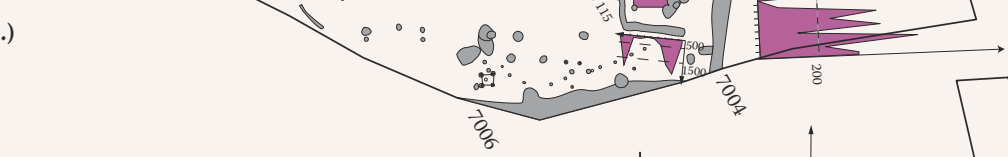

Couche 2 (moy.)

Couche 3 (inf.)

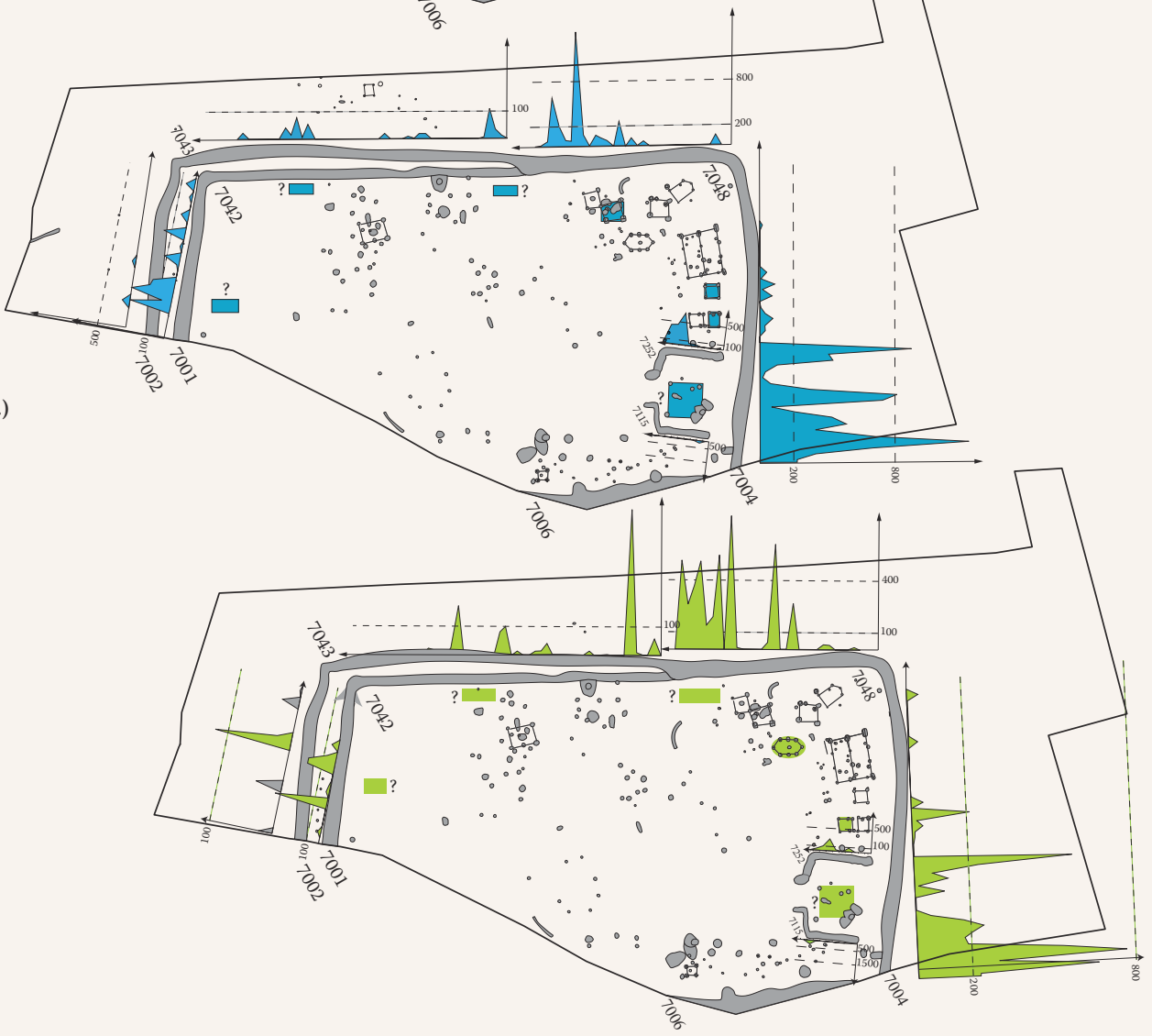



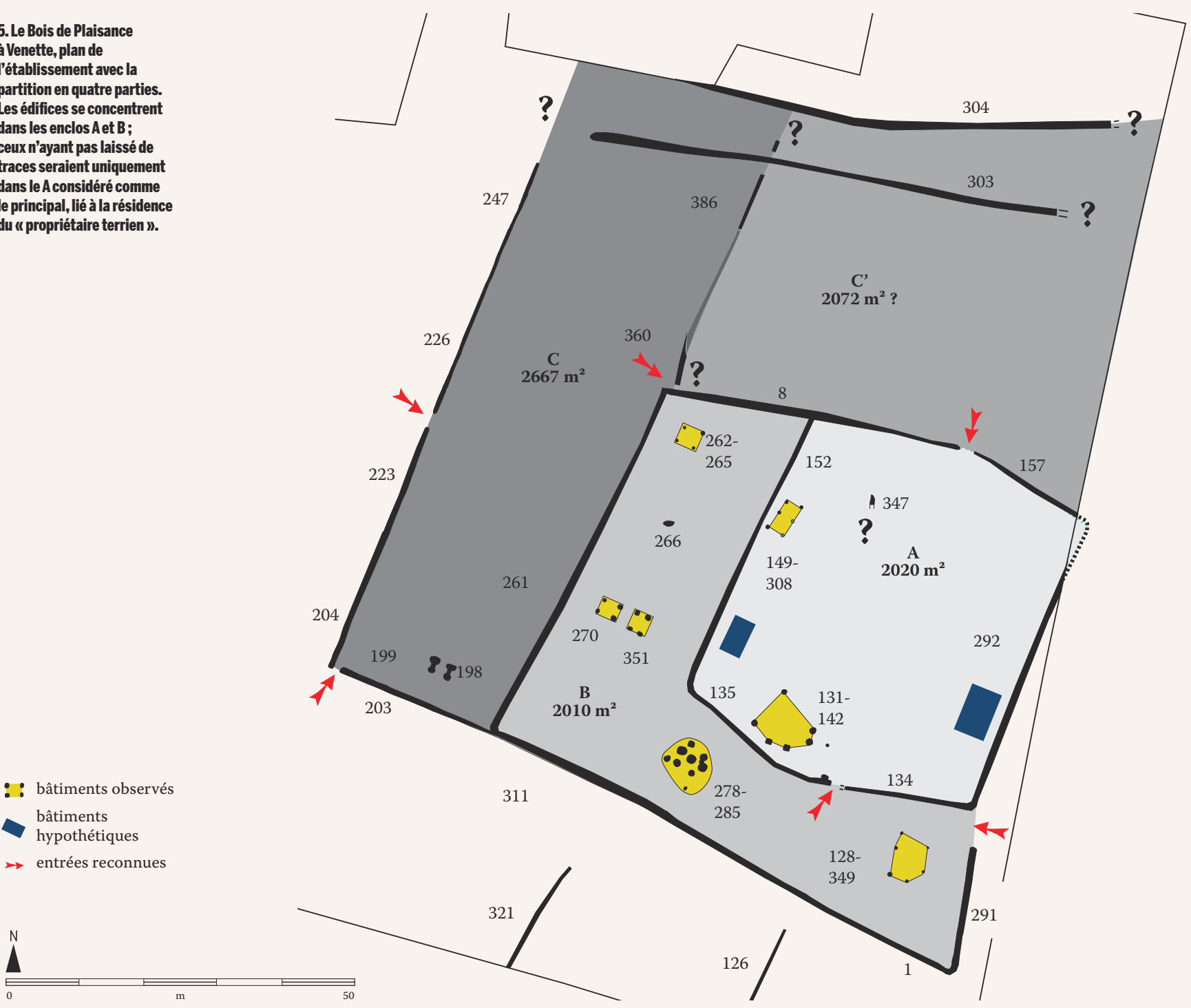

enclos, peut s'assimiler à une maison au vu des déchets perçus dans les fossés le bordant (fossés 1 et 291), alors que le bâtiment 131 est lié à de faibles amas d'artefacts. En revanche, ce dernier concentre la majorité des éléments liés à la meunerie ( $88 \%$ du poids des meules dont une dans un trou de poteau et deux dans le fossé adjacent).

La carte de répartition du grès, du torchis et des autres pierres, associée à celle des objets travaillés, donne l'occasion de visualiser deux zones de concentrations placées en vis-à-vis [ill. 6]. Celle située à l'ouest (fossé 152) se développe sur une vingtaine de mètres avec deux pôles extrêmes, tandis que celle à l'est se poursuit sur toute la longueur du fossé (292) avec un pic juste au milieu. En outre, c'est dans cette zone que l'on a trouvé un petit polissoir en grès fin, le seul bracelet en tôle de bronze et un objet énigmatique à base de sept fines plaques de fer de tailles décroissantes possédant un clou central (spectre ?). La confrontation avec le corpus céramique étudié par Muriel Friboulet confirme que ce fossé fournit l'ensemble numérique le plus important du site et le vaisselier le plus diversifié. Il faut donc considérer qu'une construction sur fondation légère était implantée en position centrale près de ce fossé 292 et qu'il pourrait s'agir de la résidence principale.

\section{Les sites antiques}

Avec la romanisation, survenue localement entre - $30 / 20$ et $30 / 40$, il semble que l'organisation spatiale et la gestion des déchets changent. En effet, la grande majorité des fermes antiques ont une durée d'occupation qui oscille entre 150 et 250 ans (Maréchal, 2009; Malrain et al., 2017). Les fossés sont régulièrement curés et certains déchets n'y sont plus rejetés, probablement évacués sous forme de fumure ou d'épandage. La perduration sur une longue durée de l'habitat se décèle sur le terrain aux nombreux recoupements, et souvent également aux nuages de trous de poteau qui doivent correspondre à de multiples (re)constructions. La moindre présence de rebus et le « désordre » apparent 


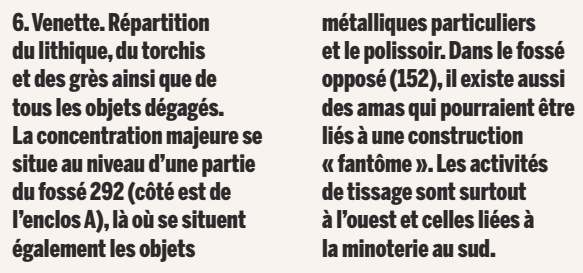

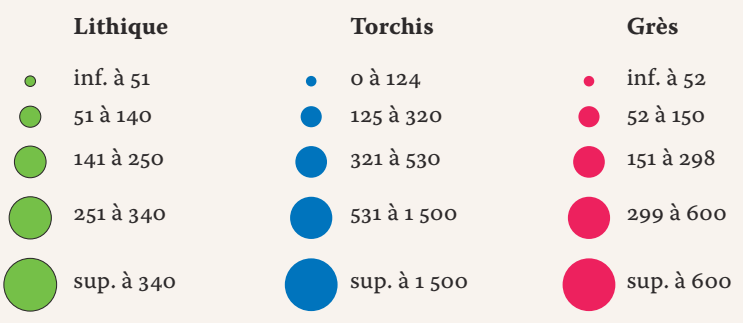

N

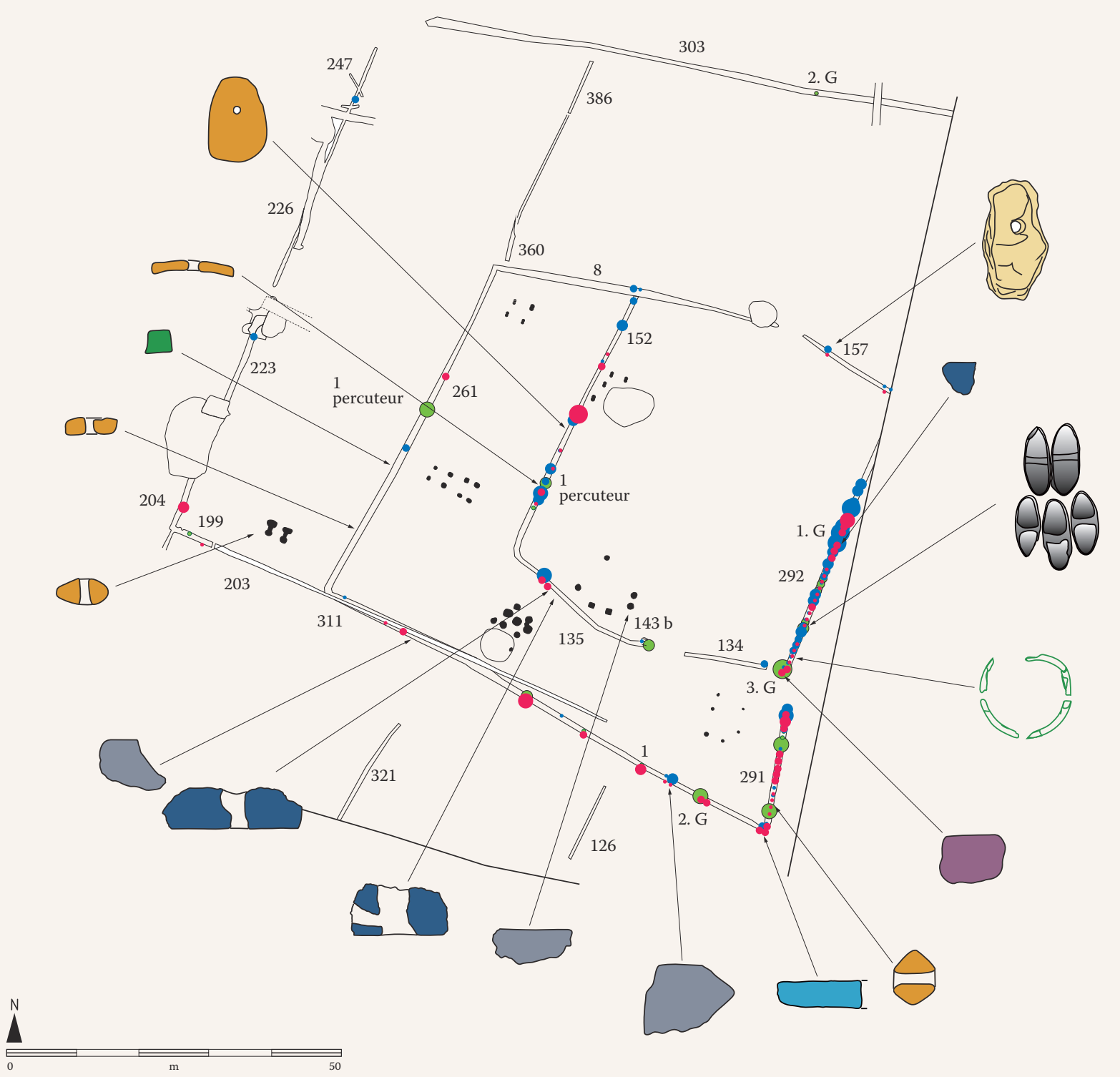

( $\mathrm{G}=$ Galet $)$
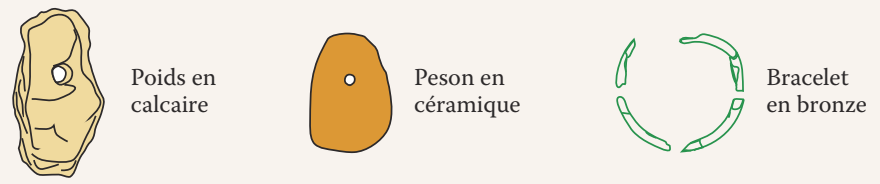

Objet en

bronze (dé ?)

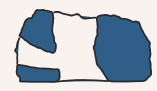

Meule (broyon)

en poudingue

ID Objet en fer énigmatique

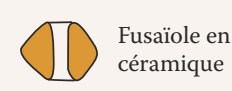

Meule en grès

Polissoir en grès très fin

Pierre à aiguiser 


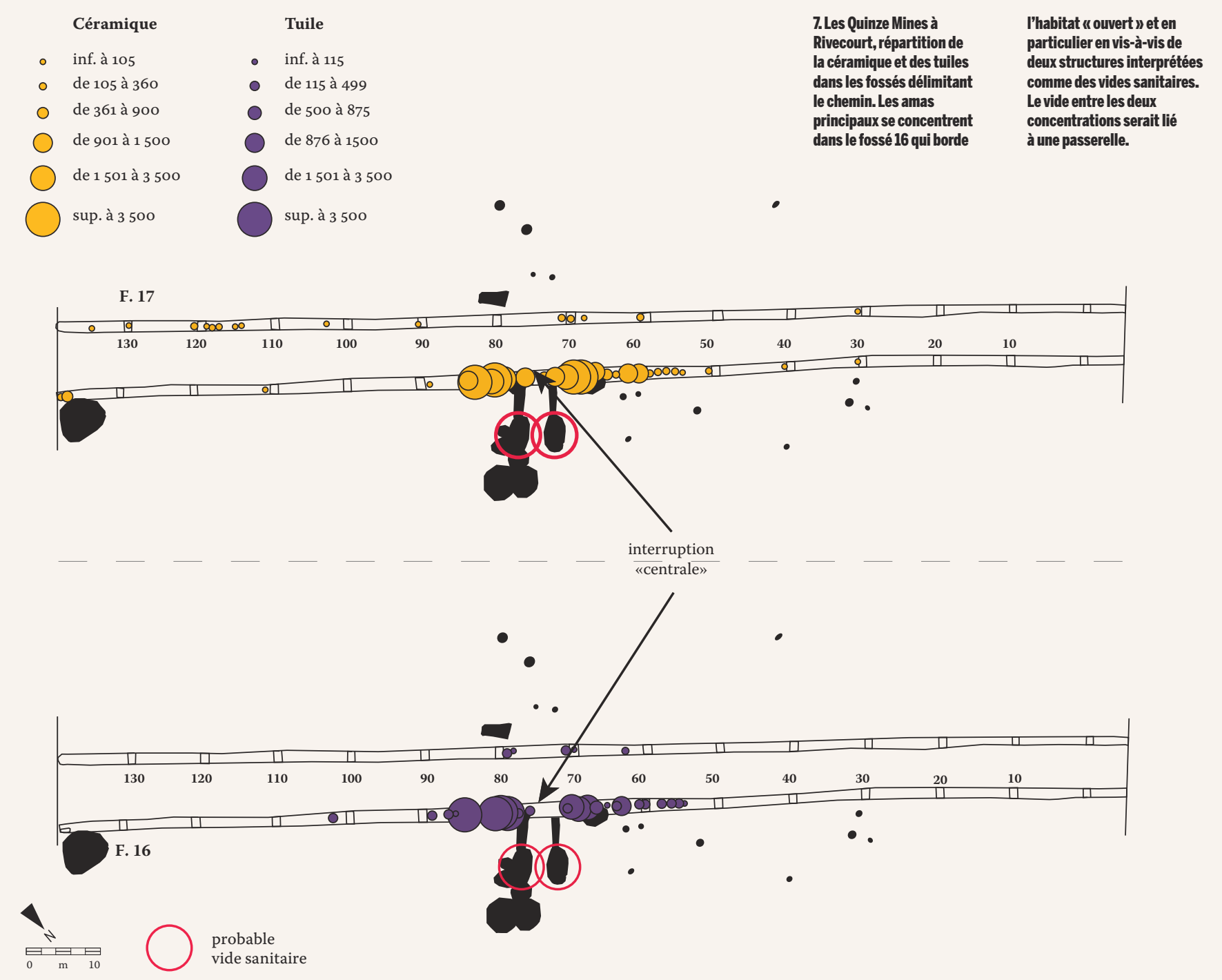

\section{న}

3. Fouillé en 2003 sous la responsabilité de l'auteur.

4. Fouillé en 2005 sous la responsabilité de l'auteur. des traces de structures rendent plus ardue l'interprétation des sites.

Sur la butte sableuse des Quinze Mines à Rivecourt, le décapage sur 2,04 ha a mis en évidence un habitat « ouvert » ayant une courte durée de vie ${ }^{3}$ (dernier tiers du I ${ }^{\mathrm{er}}-1^{\mathrm{re}}$ moitié du $\mathrm{II}^{\mathrm{e}}$ siècle). L'occupation est contiguë à un chemin délimité par deux fossés (Maréchal, 2008). Le segment de fossé qui longe l'installation fournit des amas de mobilier sur une trentaine de mètres de long, juste en vis-à-vis de l'habitat qui s'organise selon deux probables vides sanitaires [ill. 7]. Il faut noter aussi qu'il existe une interruption « centrale » dans l'amas, large de $3 \mathrm{~m}$, qui doit correspondre à la passerelle enjambant le fossé.

Sur l'établissement de la Saule Ferrée à Rivecourt ${ }^{4}$, la durée d'occupation dépasse 250 ans, mais le fossé $n^{\circ} 200$ - daté de 30/50 - n'a pas été curé après son abandon et constitue donc un ensemble clos (Maréchal, 2017). Sa fouille a mis en évidence une concentration principale - longue de $20 \mathrm{~m}$ - au milieu du fossé avec un point médian prédominant [ill. 8]. Des espaces vides bordent cet amas. L'hypothèse d'une maison sur fondation légère positionnée au milieu semble donc très crédible. Ce positionnement axial au niveau de la première cour préfigurerait le plan de la villa (Collart, 2014, p. 83-84).

Pour la période antique, il existe des structures excavées mieux préservées qui évoquent des architectures disparues de maisons : caves, celliers et vides sanitaires. Le distinguo entre ces derniers et certaines fosses de stockage ou d'atelier est parfois délicat, bien que les caractéristiques du vide sanitaire restent qu'il occupe une plus grande surface, mais sur une plus faible profondeur. Peu de sites locaux possèdent à la fois ces structures et, au-dessus, les vestiges de l'habitat; on citera l'exemple de la Plaine de Saint-Germain à Verberie et de la villa du Bufosse à Verneuil-en-Halatte (Collart, 1996 ; Fémolant, Malrain, 1996, p. 50-51). Un tableau croisé des principaux habitats sur lesquels des données sont disponibles des trois catégories d'aménagements et des niveaux 


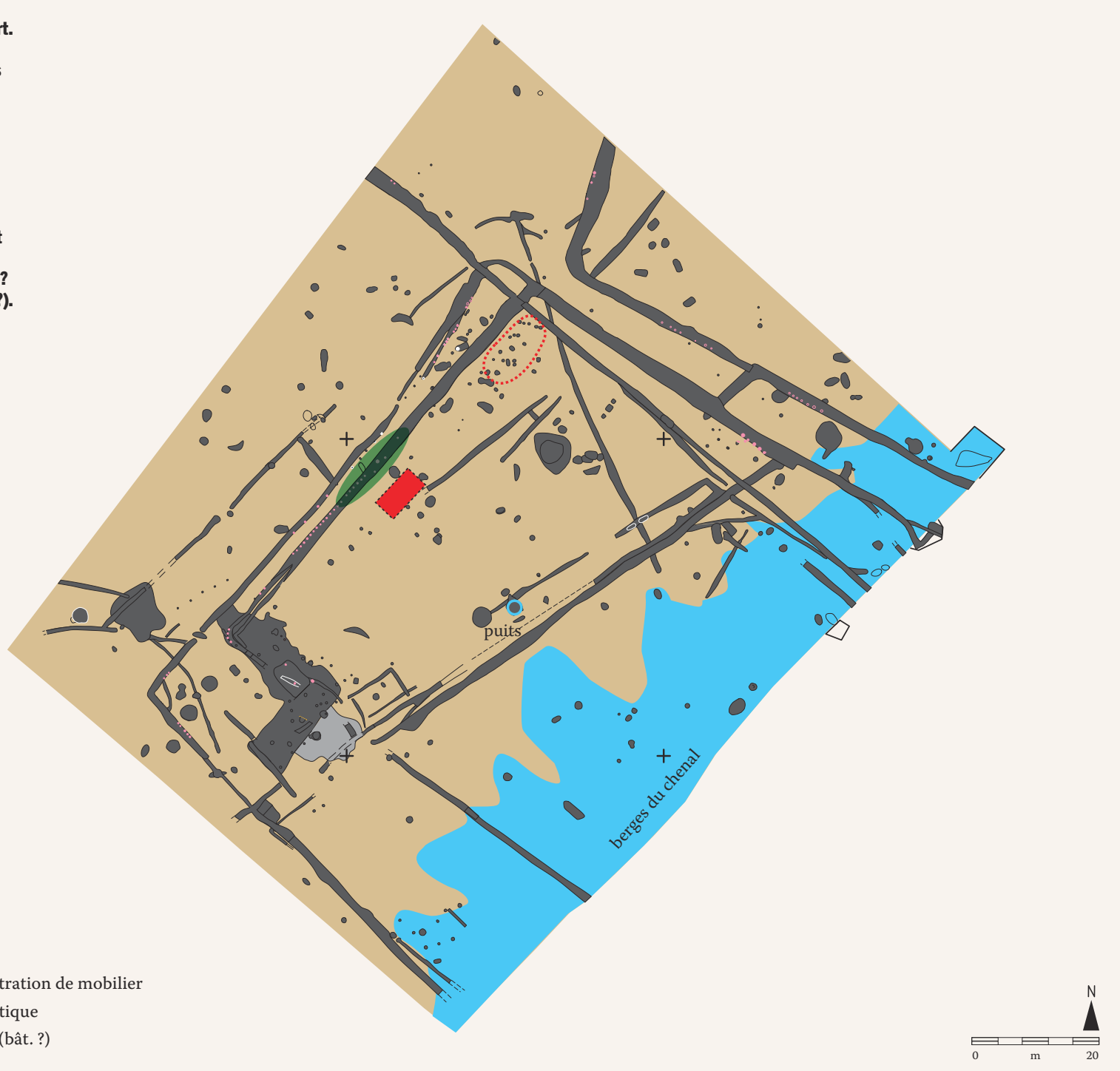

\footnotetext{
chablis

principale concentration de mobilie

bâtiment hypothétique

concentration Tp (bât. ?)
}

5. Fouillé en 1999 sous la responsabilité de Pascal Quérel, Inrap. hiérarchiques donne quelques pistes

d'interprétation [ill. 9]. Les caves ne concernent que les rangs supérieurs (1 à 3). Si leurs chronologies couvrent la période du $\mathrm{I}^{\mathrm{er}}$ au $\mathrm{III}^{\mathrm{e}}$ siècle, il faudrait opérer une distinction entre petites et grandes surfaces (sup. $10 \mathrm{~m}^{2}$ ). Ces dernières, plus rares, semblent caractériser les villae. Notons que des sites de rang 2 n'en disposent pas. Les celliers demeurent plus rares et leur chronologie se limite aux I ${ }^{\mathrm{er}}$ et $\mathrm{II}^{\mathrm{e}}$ siècles (Malrain et al., 2017, p. 312). Les vides sanitaires sont eux plus largement répandus, présents du $\mathrm{I}^{\mathrm{er}}$ au $\mathrm{III}^{\mathrm{e}}$ siècle (Ibid.). Il convient d'observer que les fermes modestes disposent rarement de ces aménagements. Les agglomérations rurales en sont également dépourvues, ce qui n'est pas le cas de l'agglomération secondaire d'Estrées-Saint-Denis ${ }^{\mathbf{5}}$ (Quérel, 2002). Sur ce site, il convient de souligner la mise en évidence de constructions tardives conservées. Les solins utilisent « des blocs massifs de grès ou de calcaire (...) les intervalles sont colmatés avec des blocs de plus petite dimension installés avec un certain soin » (Ibid., p. 303-304).

Cette observation est importante car on remarque régulièrement dans nos contextes ruraux, en relation avec des aménagements de type vides sanitaires, de grosses quantités de pierres - parfois chauffées -dans le comblement secondaire ou final (Maréchal, 2011c). Vu l'absence d'architectures reconnues et considérant les phénomènes taphonomiques, nous nous demandons si ces quantités de blocs de grès ou calcaire ne sont pas liés au démantèlement de ces solins légers. Cette architecture, plus fragile que les solins en craie, ne laisserait aucune trace. Après l'abandon, les blocs peuvent être récupérés ou rejetés, les labours vont les disperser aisément et le décapage éliminera les derniers éléments. En dehors des contextes « urbains », il faudra donc à l'avenir s'attacher à mettre en évidence ces fugaces solins non maçonnés. 
9. Tableau synthétique sur l'absence et/ou la présence des caves, celliers et vides sanitaires, confrontée aux rangs hiérarchiques (d'après Malrain et al.,2017) des habitats ruraux gallo-romains fouillés dans la moyenne vallée de l'Oise. Le facteur surface pour les caves permettrait d'opérer une sélection supplémentaire $X=$ présence; () nb; HC = hors catégorie (habitat groupé : village, hameau).

\begin{tabular}{|c|c|c|c|c|c|c|}
\hline site & qualif. & durée occup. & rang hiérar. & cave & cellier & vide sanit. \\
\hline Verneuil-en-Halatte «le Bufosse» & villa & 350 ans & 1 & 2 & & 1 \\
\hline Longueil-St-Marie « Gros Grès » IV & ferme/relais & 300 ans & 2 & & & $x$ \\
\hline Rivecourt « Petit Pâtis » & ferme & 280 ans & 2 & & & \\
\hline Venette «Bois de Plaisance» 05 & petite villa & 250 ans & 2 & 3 & 1 & \\
\hline Venette «Bois de Plaisance» 10 (diag) & ferme/relais & 250 ans & 2 & 4 & 1 & 1 \\
\hline Chevrières «Bois Madame» & ferme & inf. 1 siècle & 3 & & & 1 \\
\hline La Croix-St-Ouen «les Jardins» & ferme & 300 ans & 3 & & & \\
\hline Houdancourt « Pont à Brebis » & ferme & 250 ans & 3 & 1 & & \\
\hline Longueil-St-Marie «le Barrage» & ferme & 250 ans & 3 & & 1 & \\
\hline Longueil-St-Marie «Butte de Rhuis» & ferme & 250 ans & 3 & 1 & & \\
\hline Pont-St-Maxence « Grand Bosquet » & ferme & 250 ans & 3 & & & \\
\hline Pontpoint «le Jonquoire» & ferme & 250 ans & 3 & 1 & & \\
\hline Venette «Bois de Plaisance» 04 & ferme & 250 ans & 3 & 4 & & 1 \\
\hline Verberie «Plaine de St-Germain» & ferme & 150 ans & 3 & 2 & & \\
\hline Verberie « les Gâts » I 98 & ferme & 200 ans & 3 & & & 1 \\
\hline Verberie « les Gâts » II 98 & ferme & inf. à 1 siècle & 3 & & & 1 \\
\hline Rivecourt «Quinze Mines » & ferme & inf. à 1 siècle & 4 & & & 2 \\
\hline Pontpoint «Fond de Rambourg » & ferme & 200 ans & 4 & & & \\
\hline Verberie « les Gâts » 97 & ferme & inf. à 1 siècle & 4 & & & \\
\hline Longueil-St-Marie « Bois Harlé/Queue de Rivecourt » & village & 250 ans & $\mathrm{HC}$ & & & \\
\hline Longueil-St-Marie «Près des Grisards » II & hameau & 250 ans & $\mathrm{HC}$ & & & \\
\hline
\end{tabular}

9

\section{Références bibliographiques}

Collart J.-L., 1996, « La naissance de la villa en Picardie : la ferme gallo-romaine précoce ", in Bayard D., Collart J.-L. (dir.), De la ferme indigène à la villa romaine. La romanisation des campagnes de la Gaule, Revue archéologique de Picardie n spécial 11/1996, p. 121-156.

Collart J.-L., 2014, " Fermes et villae », in Gauthier G. (dir.), Roger Agache, détective du ciel. Découverte de l'archéologie aérienne, Vendeuil-Caply, Musée archéologique de l'Oise, p. 83-93.

FÉmolant J.-M., Malrain F., 1996, «Les établissements ruraux du deuxième Âge du Fer et leur romanisation dans le département de l'Oise ", in BAYARD D., Collart J.-L. (dir.), De la ferme indigène à la villa romaine. La romanisation des campagnes de la Gaule, Revue archéologique de Picardie n spécial 11/1996, p. 39-53.

Gransart F., Pommepuy C., 2005, « Bazochessur-Vesles "les Chantraines" (Aisne). Présentation préliminaire de l'établissement aristocratique de La Tène D1 », in Auxiette G., Malrain F. (dir), Hommages à Claudine Pommepuy, Revue archéologique de Picardie n ${ }^{\circ}$ spécial 22/2005, p. 193-216.

Malrain F., 1990, Les fermes indigènes de Chevrières «la Plaine du Marais » (Oise), Mémoire de maîtrise, Université de Paris I, 2 vol., 220 p.

Malrain F., 200o, Fonctionnement et hiérarchies des fermes dans la société gauloise du III siècle à la période romaine: l'apport des sites de la moyenne vallée de l'Oise, Thèse de doctorat sous la direction de O. Buchsenschutz, Université de Paris I, 436 p.

MALrain F., 2006, « organisation Intra-sites de La Tène ancienne à La Tène finale » et « Les critères qualitatifs », in Malrain F., Pinard E. (dir.), Les sites laténiens de la moyenne vallée de l'Oise $d u v^{e}$ au $I^{\text {er }}$ siècle avant notre ère, Revue archéologique de Picardie nº́spécial 23/2006, p. $75-98 ; 143-146$.
Malrain F., MARÉCHAL D., 2012, «Espaces ruraux dans la moyenne vallée de l'Oise : limites et perspectives », in Carpentier V., MarCigny C. (dir.), Des hommes aux champs. Pour une archéologie des espaces ruraux du Néolithique au Moyen Âge, Actes de la table ronde de Caen, tenue les 8 et 9 octobre 2008, «Archéologie \& Culture », Rennes, Presses universitaires de Rennes, p. 367-39o et pl. XLV-XLVI.

Malrain F., Pinard E., Maréchal D., 2006, « Des sites et des paysages ", in Malrain F., Pinard E. (dir.), Les sites laténiens de la moyenne vallée de l'Oise du $V^{e}$ au $I^{e r}$ siècle avant notre ère, Revue archéologique de Picardie nº́spécial 23/2006, p. 46-51.

Malrain F., Maréchal D., De Muylder M., Lepetz S., Méniel P., ZeCh-Matterne V., 2017, «Chapitre 8: La vallée de l'Oise », in REDDÉ M. (dir.), Gallia Rustica, Les campagnes du nord-est de la Gaule de la fin de l'âge du Fer à l'Antiquité tardive, Bordeaux, Pessac, Ausonius éditions, vol. 1, p. 287-337.

MARÉCHAL D., 2005, «La moyenne vallée de l'Oise: Échelles d'analyses possibles et premiers résultats de l'occupation et de la gestion des sols durant le Haut-Empire », in Petit C. (dir.), Occupation, gestion et paléoenvironnement des plaines alluviales de l'âge du Fer à l'Antiquité, Actes de la table-ronde de Molesmes (21), 17-18 septembre 1999, Presses universitaires Franc-Comtoises, Collection des Annales littéraires, p. 173-196.

MARÉCHAL D., 2008, «Le Mesnil-en-Thelle, LongueuilSainte-Marie, Rivecourt », in QUÉREL P. (dir.) Chemins, gués et établissements routiers dans l'ouest de la Gaule Belgique, Revue archéologique de Picardie, $\mathrm{n}^{\circ}$ 3/4, p. 96-100.

MARÉCHAL D., 2009, « Le village de Longueuil-SainteMarie et les autres formes d'implantation dans la moyenne vallée de l'Oise ", in LeveAu P. et al. (éd.), Les formes de l'habitat rural gallo-romain.

Terminologies et typologies à l'épreuve des réalités archéologiques, Actes du VIII ${ }^{\mathrm{e}}$ colloque AGER (Toulouse, 22-24 mars 2007), Bordeaux, Aquitania, «Aquitania supplément » 17, p. 201-217.
Maréchal D. (dir.), 2011a, Venette, Oise (Picardie). $Z A C$ du Bois de Plaisance. Les fermes gauloise et gallo-romaine, rapport d'opération, InrapSRA Picardie, $214 \mathrm{p}$.

Maréchal D. (dir.), 2011b, Venette, Oise (Picardie). $Z A C$ du Bois de Plaisance. Zone 4. L'établissement gallo-romaine, rapport d'opération, InrapSRA Picardie, $146 \mathrm{p}$.

Maréchal D. (dir.), 2011c, Attichy, l'Avenue (Oise), rapport d'opération, Inrap-SRA Picardie, 104 p.

MaréCHAL D. (dir.), 2014, Rivecourt « le Petit Pâtis » (Oise). Occupations néolithiques et protohistoriques. Établissement de La Tène $C_{2} / D_{1}$, rapport d'opération, Inrap-SRA Picardie, $183 \mathrm{p}$.

MaréChal D. (dir.), 2017, Rivecourt (Oise) «la Saule Ferrée ». Occupation Néolithique. Établissement Haut-Empire, rapport d'opération, Inrap-SRA Picardie, $320 \mathrm{p}$.

Pinard E., Collart J.-L., Malrain F., Maréchal D., 1999, « De l'architecture à la hiérarchisation sociale du $v^{\mathrm{e}}$ av. J.-C. au III ${ }^{\mathrm{e}}$ apr. J.-C. dans la moyenne vallée de l'Oise », in Braemer F., Cleuziou S., Coudart A. (dir.), Habitat et société, Actes des

$\mathrm{XIX}^{\text {es }}$ Rencontres internationales d'Antibes (22-24 oct. 1998), Antibes, APDCA, p. 363-382.

QUÉREL P., 2002, «L'habitat. L'intervention archéologique et les phases d'occupation », in Quérel P., Woimant G.-P. (dir.), Sanctuaire et habitat gallo-romains à Estrées-Saint-Denis (Oise), Revue archéologique de Picardie, nº 3/4, p. 279-319. 